\title{
VegIoT Garden: a modular IoT Management Platform for Urban Vegetable Gardens
}

\author{
Gaia Codeluppi, Antonio Cilfone, Luca Davoli, and Gianluigi Ferrari \\ Internet of Things (IoT) Lab, Department of Engineering and Architecture \\ University of Parma \\ Parma, Italy \\ \{gaia.codeluppi, antonio.cilfone, luca.davoli, gianluigi.ferrari\}@unipr.it
}

\begin{abstract}
Nowadays, the agricultural sector is facing challenges especially because of an extensive range of grueling trends. In this context, new highly technological applicationssuch as Internet of Things (IoT), Precision Agriculture (PA), and blockchain-are enabling Smart Agriculture (SA), which holds the promise to support future needs. In this extended abstract, a low-cost, modular, and energy-efficient IoT platform for SA, denoted as VegIoT Garden, based on CommercialOff-The-Shelf (COTS) devices, adopting short- and long-range communication protocols (IEEE 802.11 and LoRa), and aiming at enhancing the management of vegetable gardens through the collection, monitoring, and analysis of sensor data, related to relevant parameters of growing plants (i.e., air and soil humidity and temperature), is presented. The infrastructure is completed with an Internet-enabled Home Node (HN) and an iOS-based mobile App, developed in order to simplify data visualization and plants' status monitoring. The proposed IoT system has been validated in a real scenario (a vegetable garden) for more than a week: the collected data highlighted possible causes for a disease contracted by vegetables (namely, tomato's blossom-end root), thus validating VegIoT Garden.

Index Terms-Internet of Things; Smart Agriculture;
\end{abstract} IEEE 802.11; LoRa; CoAP; WSN; Heterogeneous Networks

\section{INTRODUCTION}

Modern farming and agricultural production processes are nowadays undergoing a massive technological transformation, in which terms such as Agriculture 4.0 and Smart Agriculture (SA) are emerging to identify this trend. A large number of innovative technologies, such as Unmanned Aerial Vehicles (UAVs), autonomous tractors, and satellite systems, will be involved in SA-oriented future scenarios. In particular, the joint adoption of both Precision Agriculture (PA) and Internet of Things (IoT) technologies will play a crucial role for modern farmers [1]. Indeed, in order to efficiently face some future trends, such as world population growth, climate changing, and natural resources depletion, as highlighted by several governmental organizations [2] — a fully-optimized and more sustainable agricultural production system has to be reached. Therefore, research and technological innovation are seen as the keys to face these challenges. In fact, the adoption of Internet of Things (IoT)-related technologies (e.g., microcontrollers/sensors/actuators with wireless communication capabilities): (i) allows to collect, fuse and analyze agricultural data coming from crops, animals, and greenhouses (e.g., soil quality, irrigation level, presence of pests, greenhouse air humidity, etc.); (ii) enables the connectivity among all SAoriented emerging technologies, thus supporting integration at different layers; and (iii) strengthens food safety and supply chain traceability. In turn, this last objective can be accomplished with various technologies, among which a promising one is the blockchain [3].

Considering this perspective, a scalable, modular and lowcost IoT/SA-oriented management platform, denoted as VegIoT Garden and aiming at supporting the farmer in improving the produced goods, identifying critical issues, and solving problems regarding the irrigation and the identification of a possible correlation between climatic features, fields productivity, and crops health, is presented in this paper. More precisely, the proposed implementation of the VegIoT Garden is based on low-cost Commercial-Off-The-Shelf (COTS) devices and IoT-oriented communication protocols, including LoRa [4] (at physical layer) and Constrained Application Protocol (CoAP) [5] (at application layer).

The rest of the paper is organized as follows. Section II is dedicated to discuss related works and present a short background overview. In Section III, the proposed architecture is described, with focus on the considered technologies. Section IV is devoted to the experimental results analysis, while a discussion, in terms of collected data and energy efficiency, is presented in Section V. Finally, in Section VI we draw some conclusions.

\section{RELATED WORKS AND BACKGROUND}

Nowadays, there is a great interest in supporting the agricultural technological transformation through the deployment of solutions focused on the most disparate technologies, among which the IoT finds applicability in a large set of possible SA-oriented applications [1]. As an example, crop monitoring involves the observation, collection, and analysis of data related to parameters which influence crops' life-cycle (e.g., soil composition, acidity, temperature, and weather conditions), with the aim of ensuring a correct growth for crops and, consequently, a satisfactory farm production. Proper adoption of IoT technologies, such as sensors, constrained devices, network infrastructures, and cloud platforms, allows to measure, transfer and process crop-related information through the deployment of dedicated data collection and monitoring systems up to the cloud. Despite the great heterogeneity proper 
of these solutions, in many cases these are Wireless Sensor Network (WSN)-based systems [6] collecting data from crops and forwarding them to Internet-like remote servers or cloud platforms, for storage and processing purposes.

Notwithstanding the availability of the technologies described above, in some cases the agricultural fields, where the monitoring WSNs are deployed, are not covered by a reliable Internet connectivity, fundamental for transmitting data remotely (e.g., fields spread far from an available IEEE 802.11 Access Point (AP) or covered by an unstable 3G/4G cellular connectivity). A viable solution to overcome this issue is the integration of heterogeneous communication protocols, in terms of throughput, transmission ranges and power requirements, in the overall system design, as proposed in [7] and detailed in the following. First, a sort of in-site connectivity (e.g., the ZigBee protocols suite [8]) is introduced, in order to link WSN nodes together and enable information exchange. Then, the collected information is transmitted and maintained in a particular entity inside the WSN, denoted as "sink/gateway node," in charge of forwarding it to another device (namely, an IoT node), placed in a location where this latter can easily access the Internet (e.g., a node located in the farmer's house with an available IEEE 802.11 AP), and possibly provided with a communication protocol able to support long-range transmissions (e.g., LoRa [4]). Finally, the IoT node transfers data to the cloud thanks to the available Internet access.

\section{ARCHITECTURE}

As anticipated in Section I, the VegIoT platform aims at supporting the management of a SA-oriented scenario, which, in our application, is the monitoring of a small-size crop (with an area of less than $100 \mathrm{~m}^{2}$ ), such as a urban vegetable garden (or eventually a greenhouse), located in an area with no Internet connectivity available in a range smaller than $100 \mathrm{~m}$ (which is a commonality in agricultural scenarios, as explained in Section II). For the sake of completeness, it is remarked that the application scenario, in which the VegIoT platform has been installed and evaluated, obviously owns the listed characteristics, being composed of a urban vegetable garden and a "farmer's house," but further details will be given in the following.

From an architectural point of view, the VegIoT platform, as shown in Figure 1, is composed of: (i) an IEEE 802.11-based WSN, denoted as Garden Wireless Sensor Network (GaWSN), located in the vegetable garden and collecting data of interest for crops (i.e., soil/air temperature and humidity) and equipped with a border router with both Wi-Fi (towards the WSN) and LoRa interfaces (Subsection III-A); (ii) an IoT node, denoted as Home Node (HN), located into the farmer's house, which provides date/time clock synchronization features to the on-field WSN, and receives and stores collected data (Subsection III-D); and (iii) an iOS mobile App (Mobile Node, MN), for accessing data associated with the overall platform. Moreover, as anticipated, the considered scenario includes: (i) a urban vegetable garden, organized in subregions, where different kinds of vegetables (i.e., zucchini,

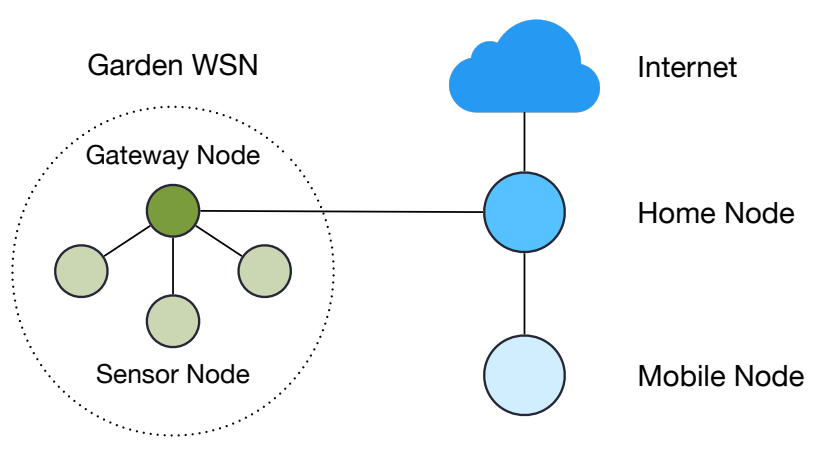

Fig. 1: VegIoT platform: main components denoting the proposed infrastructure.

tomatoes, cabbages, savoy cabbages, and beets) are grown; and (ii) the farmer's house, at approximately $200 \mathrm{~m}$ from the garden. The information exchange between GaWSN and HN relies on a bi-directional point-to-point LoRa link, since LoRa has been designed to support long-range and low-power communications, potentially involving SOs with critical constrains (e.g., in terms of memory, energy, and computational power).

\section{A. Garden WSN (GaWSN)}

The vegetable garden (with an area approximately equal to $20 \mathrm{~m}^{2}$ ) is covered by a GaWSN with a star topology, composed of three Sensor Nodes (SNs) and a Gateway Node (GN), as shown in Figure 1. The SNs, equipped with appropriate sensors, gather the following environmental parameters, which have been chosen for their relevance in all garden plants growing phases: ${ }^{1}$ (i) soil humidity and temperature; (ii) air humidity and temperature [9]. The gathered sensor data are thus sent by each $\mathrm{SN}$ to the GN, in turn internally storing them into a MongoDB database and periodically forwarding them to the HN.

The information exchange flow inside the GaWSN follows the protocol stack shown in Table I, which represents a balanced solution in terms of connectivity requirements (i.e., standardization level, lightweight and constrained SO-oriented communication).

\begin{tabular}{|c|}
\hline Application \\
\hline Transport \\
\hline Network \\
\hline Data-link \\
\hline Physical \\
\hline
\end{tabular}

TABLE I: IoT-oriented protocol stack of the designed Garden WSN (right) with corresponding reference ISO/OSI layers (left).

More precisely, the protocol suite has been defined as follows: IEEE 802.11 and Internet Protocol (IP) have been

\footnotetext{
${ }^{1}$ From here simply called "crops parameters."
} 
chosen because of their widespread adoption and compatibility with a large number of SOs (e.g., micro controllers, dailylife devices as smartphones, etc.). Furthermore, IEEE 802.11, which can be considered a quite-short-range communication protocol, has been chosen even since the VegIoT platform targets small-size crops, as mentioned before (i.e., urban vegetable gardens). Besides, CoAP and UDP have been selected because they support asynchronous and lightweight message exchange, sender and receiver decoupling, and other useful IoT-oriented features (e.g., CoAP observing mode) effective for the traffic generated by the GaWSN. Moreover, as shown in Figure 2, in the proposed VegIoT architecture the GN is also in charge of providing an IEEE 802.11-based connectivity as a dedicated AP, since the area to monitor lacks of a IEEE 802.11 coverage (or of another type of connectivity).

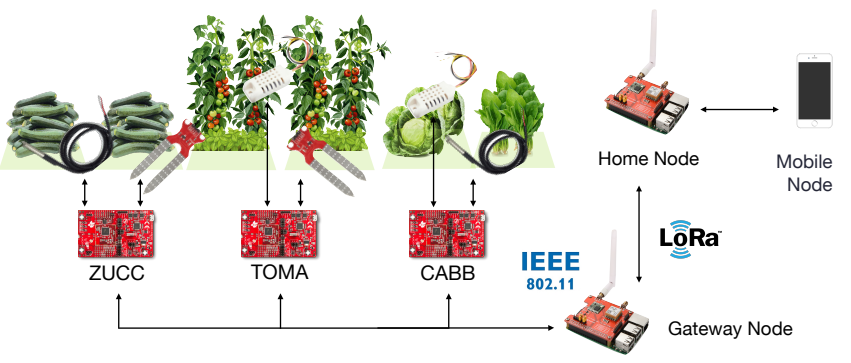

Fig. 2: VegIoT platform: devices, sensors and communication protocols deployment.

\section{B. Sensor Nodes (SNs)}

As anticipated in Section III-A, the data collected by the VegIoT SNs comes from the specific monitored vegetables, namely: tomatoes (TOMA); zucchini (ZUCC); and cabbages (CABB). In detail, each SN integrates a TI CC3200 Launchpad (denoted as CC3200, for short, in this paper) with humidity and temperature sensors (summarized in Table II), is powered by two type-AA batteries, and is encapsulated in a waterresistant box in order to be protected from potential "dangers" typical of a garden (e.g., severe weather, watering, domestic animals and insects). Although alternative commercial boards can been selected, because of its built-in IEEE 802.11 connectivity, low price and reduced dimensions, the CC3200 is an appealing choice.

\begin{tabular}{|c|c|c|c|}
\hline Node & Sensor & Collected Data & Type \\
\hline \multirow{2}{*}{ TOMA } & AOSONG AM2302 & Air temperature and humidity & Digital \\
\cline { 2 - 4 } & Soil Moisture Sensor Module & Soil humidity & Analog \\
\hline \multirow{2}{*}{ ZUCC } & DS18B20 & Soil temperature & Digital \\
\cline { 2 - 4 } & Soil Moisture Sensor Module & Soil humidity & Analog \\
\hline \multirow{2}{*}{ CABB } & AOSONG AM2302 & Air temperature and humidity & Digital \\
\cline { 2 - 4 } & DS18B20 & Soil temperature & Digital \\
\hline
\end{tabular}

TABLE II: Monitoring sensors adopted in VegIoT.

Finally, from a software perspective, each SN runs a C program based on: (i) a setup phase, executed only once at the device start-up (or reset), needed to configure all the required functionalities; and (ii) a loop phase, executed cyclically (when the setup phase is over) until the device resets or the energy depletes. We remark that, in the loop phase, the node alternates the execution of its tasks (activity period), with sleeping periods in order to save battery (exploiting the CC3200 hibernation mode). In the activity period, sensor data are captured and exposed to any clients on a CoAP server, in a JSON format, through an observable CoAP resource (in order to let clients be automatically notified when new sensor data are available).

\section{Gateway Node (GN)}

The GN provides the following functionalities: (i) data collection of the parameters measured by SNs; (ii) data storage, into a MongoDB database; (iii) periodic forwarding of the collected data to the $\mathrm{HN}$; and (iv) IEEE 802.11 AP-mode network connectivity spreading. On the architectural side, the GN is based on a Raspberry Pi (RPi) equipped with a LoRa GPS HAT, in order to create a bi-directional point-to-point LoRa link to exchange data between GN and HN.

Moreover, GN and HN share a timestamp, which is sent by the HN (connected to the Internet) to the GN with the aim of supporting GaWSN correct date/time synchronization (this is necessary in order to assign a correct date/time reference to the collected data). Despite its inherent benefits (e.g., built-in IEEE 802.11 connectivity), the RPi has a non-negligible drawback represented by its high power consumption, leading to a critical power management in battery-powered applications: this is the reason why the GN is not battery-powered.

\section{Home Node $(H N)$}

As highlighted before, the HN has been deployed with the same hardware as the GN (RPi board equipped with a LoRa GPS HAT) and is placed in the farmer's house. The HN receives sensors data forwarded by the GN, stores them into a MongoDB database, and is connected to Internet through a (wired/wireless) link with the farmer's domestic IEEE 802.11based AP. In this way, the $\mathrm{HN}$ is able to: (i) provide date/time synchronization to the GN at its system start-up (i.e., sending a UNIX-like timestamp); (ii) gather, from the Internet, other information useful for the VegIoT platform, such as weather data (e.g., pollution levels, meteorological information); and, eventually, (iii) transfer stored data to the cloud (feature already designed and to be implemented in the next release of the VegIoT architecture). Finally, the HN's stored data can be visualized on a Mobile Node (MN) using the VegIoT App, an iOS-based mobile App allowing the farmer to monitor the status of garden plants and being notified of any dangerous situations in a very intuitive way.

\section{E. Mobile Node $(M N)$}

As anticipated in Section III-D, the VegIoT App has been developed in order to simplify data visualization and vegetables status monitoring. As an example, through the MN the farmer can check if vegetables need to be watered, evaluate 
soil moisture levels through collected data, or set specific alarms (as shown in Figure 3b) by which he/she can be notified (e.g., through an e-mail, as depicted in Figure 3c) should crop's parameters reach values out of a properly set threshold. Furthermore, the MN has been written in Swift 3 language and installed and tested on an Apple iPhone 7 (equipped with iOS 12 as Operating System). The developed App retrieves the data stored into the HN database querying the CoAP server on a specific CoAP resource through the GaWSN IEEE 802.11 connection. A snapshot kept from the VegIoT App is shown in Figure 3a, in which the trend of a group of TOMA-related data is compared through a line chart.

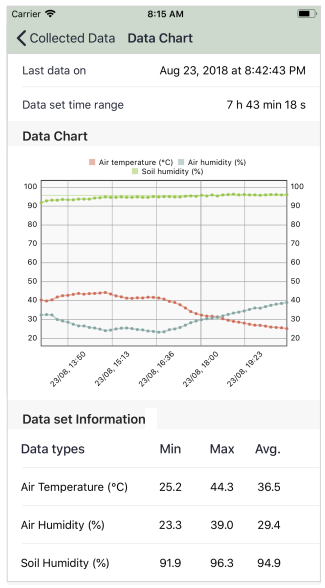

(a)

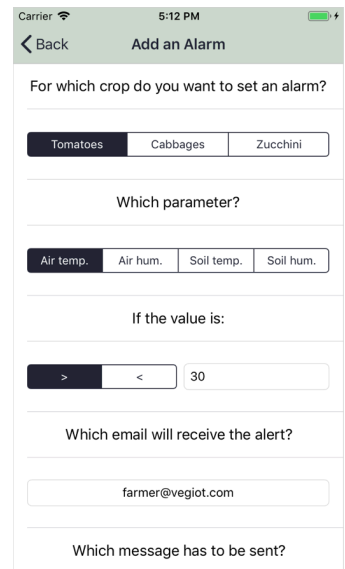

(b)

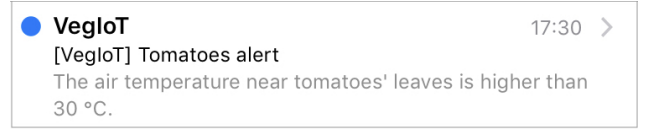

(c)

Fig. 3: VegIoT platform: mobile App snapshot, showing a group of retrieved sensor data compared through a line chart (a) and the form used to set an email alarm; and an example of an email notification.

\section{EXPERIMENTAL RESULTS}

The proposed VegIoT platform has been deployed and validated with the following phases: (i) validation of the data collection functionality and (ii) evaluation of the SNs's battery lifetime.

\section{A. Collected Data}

VegIoT has been validated gathering data on a time interval longer than one week, with a sensor sampling interval equal to $10 \mathrm{~min}$. In Figure $4 \mathrm{a}$ and Figure $4 \mathrm{~b}$, the temperature and humidity values gathered during two days of test are shown, respectively.

As can be seen, the monitored parameters seem to follow some periodic trends and relations. For instance, air temperature increases during daylight hours and decrease during night; as expected, air humidity has the opposite behaviour.
In general, soil temperature and humidity vary much less. It can be observed that the air temperature measured among tomato leaves reaches high values (higher than $30^{\circ} \mathrm{C}$ ) in the early hours of the afternoon. This environmental condition, combined with others (e.g., calcium deficit, water stress), can increase the probability that tomatoes exhibit the so-called "blossom-end root" [10], which effectively affected some of the tomatoes planted in the monitored urban vegetable garden (as shown in Figure 4c). Therefore, the use of VegIoT could have prevented this problem.

\section{B. Energetic Autonomy Analysis}

A key aspect to deal with, in the deployment of an IoT system, is related to energetic autonomy and power utilization of network nodes (i.e. SOs). Indeed, although efficient energy management is generally an important factor, it becomes extremely relevant when a system is composed of one or more battery-powered devices (as the VegIoT platform is). For this reason, we thus investigate the energetic autonomy and current consumption of the battery-operated nodes (namely, the SNs) in the VegIoT platform. In general terms, by energetic autonomy we refer to the operational autonomy of a SN, based on its battery energy and operational pattern. Otherwise, in temporal terms, the energetic autonomy corresponds to SNs lifetime.

First, the mean current consumption and time duration of SNs in their different states (activity and inactivity/sleep) have been measured and the collected experimental results are summarized in Table III.

\begin{tabular}{|c|c|c|}
\hline State & Mean current $[\mathrm{mA}]$ & Duration [s] \\
\hline Activity & $I_{\text {work }}=47.2$ & $t_{\text {work }}=9$ \\
\hline Inactivity/sleep & $I_{\text {hib }}=9$ & $t_{\text {hib }}=600$ \\
\hline
\end{tabular}

TABLE III: Experimental results on mean current consumption and time duration of a $\mathrm{SN}$ during its operational phases (activity and inactivity/sleep).

An approximate expression for the mean consumed current $I_{\text {mean }}$ can then be calculated as follows:

$$
I_{\text {mean }}=\frac{t_{\mathrm{work}} \cdot I_{\mathrm{work}}+t_{\mathrm{hib}} \cdot I_{\mathrm{hib}}}{t_{\mathrm{work}}+t_{\mathrm{hib}}} \approx 9.57 \mathrm{~mA} \text {. }
$$

Assuming that a $\mathrm{SN}$ is powered by two type-AA batteries $\left(1.5 \mathrm{~V}\right.$, LR6 model) with a per-battery capacity $C_{\mathrm{AA}}$ (dimension: [mAh]), the SN's expected lifetime (in other words, its energetic autonomy) $t_{\mathrm{SN}}$ can be calculated according to:

$$
t_{\mathrm{SN}}=\frac{2 \cdot C_{\mathrm{AA}}}{I_{\text {mean }}} .
$$

As an example, adopting two type-AA batteries with a perbattery capacity $C_{\mathrm{AA}}=2300 \mathrm{mAh}$, the theoretical expected lifetime, calculated according to Eq. (2), is about 20 days. In practice, due to the fact that the supplied potential of type-AA batteries tends to decrease with usage, the nominal capacity cannot be entirely exploited. Indeed, there is a voltage value (namely, $2.5 \mathrm{~V}$ for the $\mathrm{CC} 3200$ board) below which 


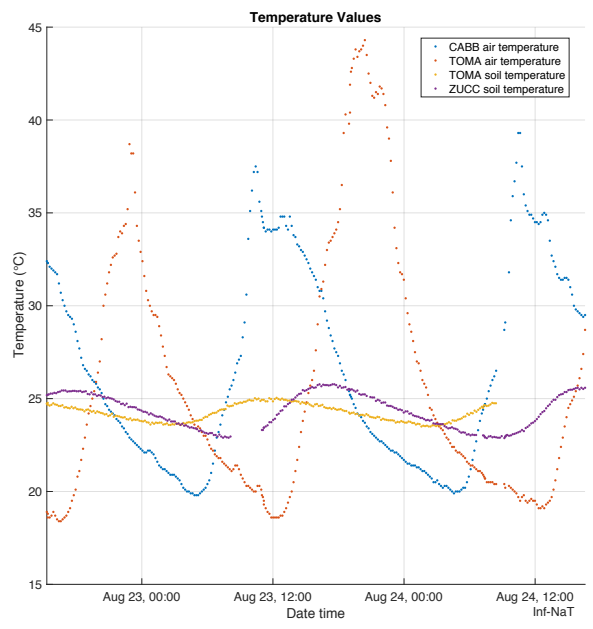

(a)

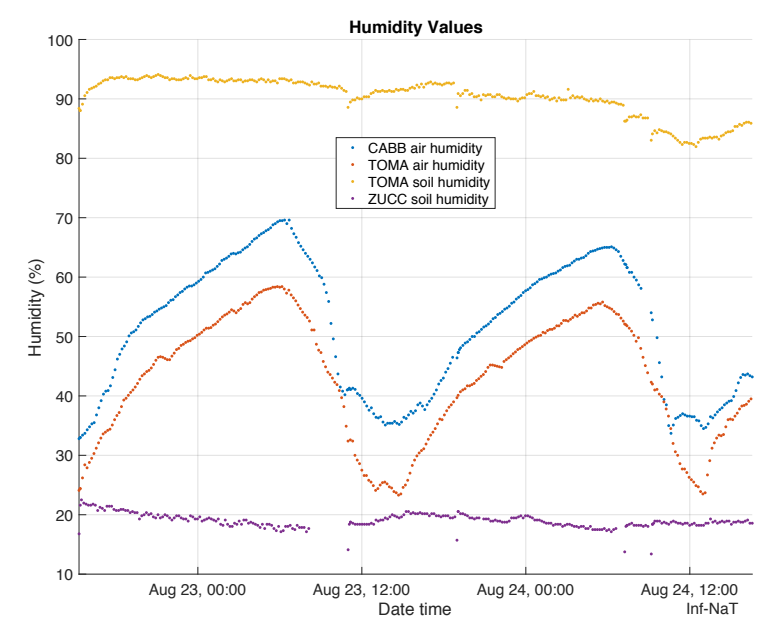

(b)
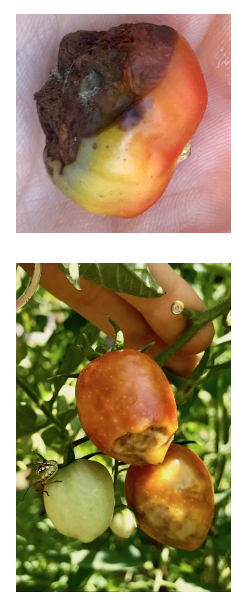

(c)

Fig. 4: Experimental results: (a) soil and air temperature values collected during two days of test; (b) soil and air humidity values collected during two days of test; (c) blossom-end root disease.

the CC3200 board stops working properly: this reduces the effective lifetime of the SN.

In order to define a more realistic lifetime expected value, the following energy discharge experiment has been performed: two alkaline batteries (namely, Panasonic Pro Power) have been chosen to power a SN until the batteries' voltage reaches a value below which the board can no longer transmit data correctly. The experimental evaluation revealed that the node autonomy is around 11 days, as will be further discussed in Subsection V-A.

\section{Discussion}

\section{A. Energetic Autonomy (Lifetime)}

As described in Section IV-B, a SN powered with two typeAA batteries can work continuously for about 11 days, i.e., 9 days less than the hypothetical duration (20 days) estimated through Eq. (2). Although this experimental result is interesting (providing the reader with a wider and more realistic point of view concerning SNs' energetic requirements), it is quite evident that, for a long-term deployment of the VegIoT system, it is mandatory to select another power source (indeed, no farmer would adopt a solution which requires him/her to change batteries every 11 days). Hence, a first consideration involves the replacement of the two type-AA batteries with a solar-panel-charged lithium battery, built, for example, using the low-cost products shown in Figure 5. As an example, a Lithium Polymer (LiPo) battery can be easily integrated with a solar panel through the usage of a "LiPo Charger Modules" (shown in Figure 5b), with the aim of building a more durable and greener power supply, thus minimizing human assistance (i.e., for changing batteries every few days).

In fact, with a small $1 \mathrm{~W}$ solar panel, and a $1800 \mathrm{mAh}$ LiPo battery, with a $3.7 \mathrm{~V}$ working tension, in a sunny day, during which the average temperature is around $25^{\circ} \mathrm{C}$, the solar panel, if correctly oriented towards the sun, will take approximately 13-14 hours to fully recharge the battery. Thus, since the estimated duration normally coincides with the number of daylight hours in the summer season, a mildly bright summer day is sufficient to fully recharge the chosen LiPo battery. Although the stated assumptions refer to an optimistic scenario, even with worse operational conditions (e.g., cloudy days, sub-optimal solar panel orientation), two or three days are usually sufficient to reach the same result. Finally, considering the amount of energy daily depleted by a SN for its activity (which is very low and approximately equal to $10 \mathrm{mAh}$ ), it can concluded that a SN can work for several months without any problem. Indeed, even if its battery fully discharges (i.e., due to multiple consecutive days of bad weather), a SN restarts to transmit when the battery reaches a sufficient charge, without any kind of manual intervention.

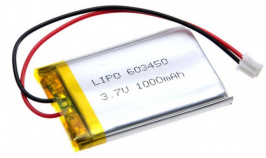

(a)

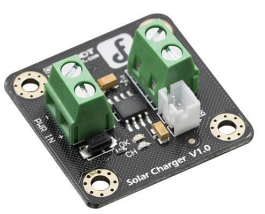

(b)

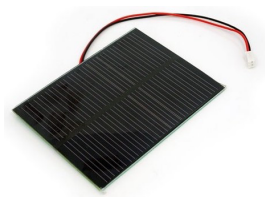

(c)
Fig. 5: Power source based on solar-panel-charged battery: (a) LiPo battery; (b) solar charger module for batteries; (c) solar panel.

A second improvement concerns the introduction of mechanisms for supervising the battery charge level of each SN. For instance, each SN can periodically send a battery status update to the GN, which, for a depletion level above a predefined threshold, can forward it to the HN. The HN can in turn notify the farmer through the VegIoT App or an e-mail. 


\section{B. Blockchain as an Enabler}

In the last years, blockchain has become more and more popular, finding always new application scenarios: one of them is the agri-food supply chain traceability [11]. Concerning the proposed VegIoT platform, since it targets a food supply chain's starting point—namely, an agricultural field—and collects information related to growing conditions of some food production's primary materials (e.g., environmental conditions used to cultivate vegetables, origin country/region of products), the blockchain can be introduced to store and trace this information, in a secure and certificated way. Even through the physical implementation of a blockchain is out of this paper's scope, we discuss on the most suitable VegIoT node candidate to be blockchain-enabled.

First, a blockchain-enabled node must fulfill crucial requirements, such as, just to name a few: robust Internet connectivity; reliable power supply; and adequate memory storage space. For this reason, the most suitable node is clearly the HN: it possesses all the cited features, in contrast to the GN and the SNs, which lack them and, thus, cannot be directly employed in the blockchain domain.

Moreover, the adequate memory storage space is a prerequisite to adhere to certain security requirements. Indeed, in a context in which the blockchain "connection" is enabled, each node has to interface with the blockchain backbone securely, through mechanisms normally requiring the storage of some data (e.g., encryption keys, digital certificates, digital identities, smart contracts keys, etc.). If these data have to be maintained by each node, it is clear that SNs cannot represent a viable solution, due to their previously described constraints.

\section{Vi. Conclusions}

The SA-oriented VegIoT platform described in this paper allows to gather, monitor, and analyze sensor data collected from an urban vegetable garden. The adoption of the proposed IoT-oriented platform improves garden management, providing the farmer with a tool to solve multiple issues and suggesting proper actions to be taken in various cultivation phases. For instance, monitoring soil moisture allows to understand when plants watering is required, while analyzing soil temperature allows to understand which is the best week to seed, as well as which seeds germination would be the fastest.

Furthermore, monitored parameters can also be visualized in order to find meaningful trends, useful in detecting (and preventing) possible crops' diseases. To do this, VegIoT is composed by a WSN (GaWSN), a gathering node (HN), and an iOS mobile App (MN). The chosen technologies have allowed to reach a fair trade-off between costs, adequate communication standardization level, good modularity degree, and scalability. This can be exploited to easily introduce new SNs and functionalities into the network.

Concerning some possible future research directions, VegIoT can be seen as (i) a powerful platform to gather data, which can be further analyzed (e.g., with Artificial Intelligence (AI)- or Machine Learning (ML)-based techniques), and (ii) an exploratory and modular platform, easily extendable with new "enablers" (e.g., blockchain). This can allow to predict farm productivity and to prevent plant diseases: for example, gathered data may be exploited to automatically predict if a plant disease is related to specific environmental conditions, as well as taking preventive measures to avoid these diseases.

\section{ACKNOWLEDGMENT}

The work of the authors is partially funded by the European Commission H2020 Framework Program, under Grant No. 783221, AFarCloud project - "Aggregate Farming in the Cloud." The work of Luca Davoli is also funded by the University of Parma, under "Iniziative di Sostegno alla Ricerca di Ateneo" program, "Multi-interface IoT sYstems for Multilayer Information Processing (MIoTYMIP)" project. The work of Gaia Codeluppi is also funded by the Regione Emilia Romagna, under "Sistemi IoT per la raccolta e l'elaborazione dei dati efficienti in agricoltura di precisione e sostenibile (AgrIoT)" Ph.D. scholarship. The work reflects only the authors' views; the European Commission is not liable for any use that may be made of the information contained herein.

\section{REFERENCES}

[1] A. Tzounis, N. Katsoulas, T. Bartzanas, and C. Kittas, "Internet of Things in agriculture, recent advances and future challenges," Biosystems Engineering, vol. 164, pp. 31-48, 2017, doi:10.1016/j.biosystemseng.2017.09.007.

[2] Food and Agriculture Organization of the United Nations (FAO), "The future of food and agriculture - Alternative pathways to 2050," http://www.fao.org/3/CA1553EN/ca1553en.pdf, Accessed: 2019-09-10.

[3] R. Casado-Vara, J. Prieto, F. D. la Prieta, and J. M. Corchado, "How blockchain improves the supply chain: case study alimentary supply chain," Procedia Computer Science, vol. 134, pp. 393-398, 2018, doi:10.1016/j.procs.2018.07.193.

[4] M. Bor, J. Vidler, and U. Roedig, "LoRa for the Internet of Things," in Proceedings of the 2016 International Conference on Embedded Wireless Systems and Networks, ser. EWSN'16. Lancaster University, 2016, pp. 361-366. [Online]. Available: http://eprints.lancs.ac.uk/77615/

[5] Z. Shelby, K. Hartke, and C. Bormann, "The Constrained Application Protocol (CoAP)," Internet Requests for Comments, Internet Engineering Task Force (IETF), RFC 7252, June 2014. [Online]. Available: https://tools.ietf.org/html/rfc7252

[6] T. Ojha, S. Misra, and N. S. Raghuwanshi, "Wireless sensor networks for agriculture: The state-of-the-art in practice and future challenges," Computers and Electronics in Agriculture, vol. 118, pp. 66-84, 2015, doi:10.1016/j.compag.2015.08.011.

[7] L. Davoli, L. Belli, A. Cilfone, and G. Ferrari, "From Micro to Macro IoT: Challenges and Solutions in the Integration of IEEE 802.15.4/802.11 and Sub-GHz Technologies," IEEE Internet of Things Journal, vol. 5, no. 2, pp. 784-793, April 2018, doi:10.1109/JIOT.2017.2747900.

[8] J. Gutiérrez, J. F. Villa-Medina, A. Nieto-Garibay, and M. A. PortaGandara, "Automated Irrigation System Using a Wireless Sensor Network and GPRS Module," IEEE Transactions on Instrumentation and Measurement, vol. 63, no. 1, pp. 166-176, Jan 2014, doi:10.1109/TIM.2013.2276487.

[9] S. Alexander, "A Guide to Vegetable Growing - 8th Edition," https://www.teagasc.ie/publications/, Accessed: 2019-04-15.

[10] P. Adams and L. C. Ho, "Effects of environment on the uptake and distribution of calcium in tomato and on the incidence of blossomend rot," Plant and Soil, vol. 154, no. 1, pp. 127-132, Jul 1993, doi:10.1007/BF00011081.

[11] A. Kamilaris, A. Fonts, and F. X. Prenafeta-BoldI, "The rise of blockchain technology in agriculture and food supply chains," Trends in Food Science \& Technology, vol. 91, pp. 640-652, 2019, doi:10.1016/j.tifs.2019.07.034. 